THE Astrophysical JOURNAL LETTERS

Preprint typeset using LATEX style emulateapj v. 08/22/09

\title{
HIGH ANGULAR RESOLUTION RADIO OBSERVATIONS OF THE HL/XZ TAU REGION: MAPPING THE 50 AU PROTOPLANETARY DISK AROUND HL TAU AND RESOLVING XZ TAU S INTO A 13 AU BINARY
}

\author{
Carlos Carrasco-GonzÁlez ${ }^{1,2}$, Luis F. RodríGuez ${ }^{2}$, Guillem Anglada ${ }^{1}$, SAlvador Curiel ${ }^{3}$ \\ (Received November 14, 2008; Accepted January 22, 2009) \\ The Astrophysical Journal Letters
}

\begin{abstract}
We present new $7 \mathrm{~mm}$ and archive $1.3 \mathrm{~cm}$ high angular resolution observations of the HL/XZ Tau region made with the VLA. At $7 \mathrm{~mm}$, the emission from HL Tau seems to be arising in a clumpy disk with radius of

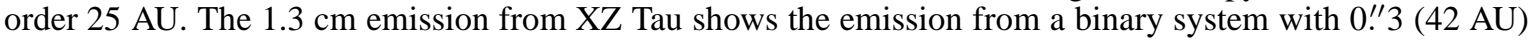
separation, known from previous optical/IR observations. However, at $7 \mathrm{~mm}$, the southern radio component

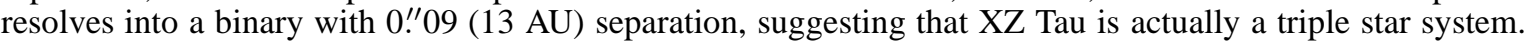
We suggest that the remarkable ejection of gas from the XZ Tau system observed with the HST may be related to a periastron passage of this newly discovered close binary system.

Subject headings: ISM: individual (XZ Tau, HL Tau) — ISM: jets and outflows — radio continuum: ISM — stars: formation
\end{abstract}

\section{INTRODUCTION}

The region encompassing the stars HL/XZ Tau, in the northeastern part of the L1551 dark cloud, has been the subject of many studies over the years. This region lies at a distance of 140 pc (e.g., Kenyon et al. 1994; Torres et al. 2009) and it is particularly rich in $\mathrm{HH}$ jets, being one of the regions where this phenomenon was first identified (Mundt \& Fried 1983).

HL Tau is one of the most intensively studied T Tauri stars. Since the first proposal that this star is associated with a nearly edge-on circumstellar disk (Cohen 1983), numerous studies have been carried out in order to image the proposed disk (e.g., Sargent \& Beckwith 1991; Wilner et al. 1996; Looney et al. 2000). This star has been proposed as the exciting source of a molecular outflow (e.g., Torrelles et al. 1987; Monin et al. 1996). HL Tau is also the source of a collimated optical jet-counterjet system that has been extensively studied (e.g., Mundt et al. 1990; Rodríguez et al. 1994; Anglada et al. 2007). Very recently, Greaves et al. (2008) presented high angular resolution maps at $1.3 \mathrm{~cm}$ of the jet-disk system in HL Tau. These authors reported the detection of a weak 1.3 $\mathrm{cm}$ source which they propose is a $14 \mathrm{M}_{J}$ proto-planet orbiting at a radius of $\sim 65 \mathrm{AU}$ around $\mathrm{HL}$ Tau.

XZ Tau, located $\sim 25^{\prime \prime}$ to the east of HL Tau, is a close binary system composed of a T Tauri star and a cool companion separated by $0 .{ }^{\prime \prime} 3$ (Haas et al. 1990). XZ Tau is also the source of an optical outflow, as revealed, e.g., by the studies of Mundt et al. (1990). The spectacular sequence of Hubble Space Telescope (HST) images of Krist et al. (1999) shows evidence of the expansion of nebular emission, moving away from XZ Tau with a velocity of $\sim 70 \mathrm{~km} \mathrm{~s}^{-1}$. This bubble of nebular emission is different from the collimated jets seen in other young stars, for example HL Tau. Recent new HST observations (Krist et al. 2008) revealed a succession of bubbles and a fainter counterbubble. In addition, they reveal that both

\footnotetext{
${ }^{1}$ Instituto de Astrofísica de Andalucía (CSIC), Camino Bajo de Huétor 50, E-18008 Granada, Spain; charly@iaa.es, guillem@iaa.es

${ }^{2}$ Centro de Radioastronomía y Astrofísica (UNAM), Apartado Postal 3-72 (Xangari), 58089 Morelia, Michoacán, México; 1.rodriguez@astrosmo.unam.mx

${ }^{3}$ Instituto de Astronomía (UNAM), Apartado Postal 70-264, D.F. 04510, Mexico; scuriel@astroscu.unam.mx
}

components of the XZ Tau binary are also driving collimated jets. Krist et al. (2008) proposed that the bubble is the result of a large velocity pulse in the collimated jet driven by the southern component of the XZ Tau system.

In this Letter, we present the results of sensitive highangular resolution Very Large Array (VLA) observations at $1.3 \mathrm{~cm}$ and $7 \mathrm{~mm}$ of HL/XZ Tau. These observations allowed us to map the dust emission from the disk of HL Tau at scales of $\sim 7 \mathrm{AU}$ and reveal that XZ Tau is actually a triple-star system with the southern optical component resolved into a close binary with a separation of only $13 \mathrm{AU}$.

\section{OBSERVATIONS}

Observations at $7 \mathrm{~mm}$ continuum were made using the VLA of the National Radio Astronomy Observatory (NRAO) ${ }^{4}$. The data were taken in $\mathrm{A}, \mathrm{B}$ and $\mathrm{D}$ configurations (VLA Project Codes: AC0763, AC0850 and AC0816). Total on-source integration times were $\sim 3 \mathrm{~h}$ at A Configuration (observed in three runs during 2004 October 15, 17 and 26), $\sim 1 \mathrm{~h}$ at B Configuration (2006 September 19), and $\sim 1.5 \mathrm{~h}$ at D Configuration (observed in two runs during 2006 January 20 and 22). The fast-switching technique was used for all the observations. Phase and flux calibrators were $0431+175$ and 3C286, respectively. The same phase center was used for all the observations.

We also used archive continuum data at $1.3 \mathrm{~cm}$ taken with the VLA in its A Configuration (VLA Project Code: AG0711) with a total on-source integration time of $\sim 12 \mathrm{~h}$ (observed in two runs during 2006 March 12 and April 1). Phase and flux calibrators were 0431+206 and 3C286, respectively. The Pie Town antenna was used originally in these observations, but we did not include it in our analysis.

Data editing and calibration were carried out using the Astronomical Image Processing System (AIPS) package of NRAO, following the standard high-frequency VLA procedures. Primary beam corrections were applied using the task PBCOR of AIPS.

Maps at $7 \mathrm{~mm}$ were made by concatenating the data from all the configurations (A, B and D). This allowed us to improve by $\sim 20 \%$ the signal-to-noise ratio of the A configuration data

\footnotetext{
${ }^{4}$ The NRAO is a facility of the National Science Foundation operated under cooperative agreement by Associated Universities, Inc.
} 


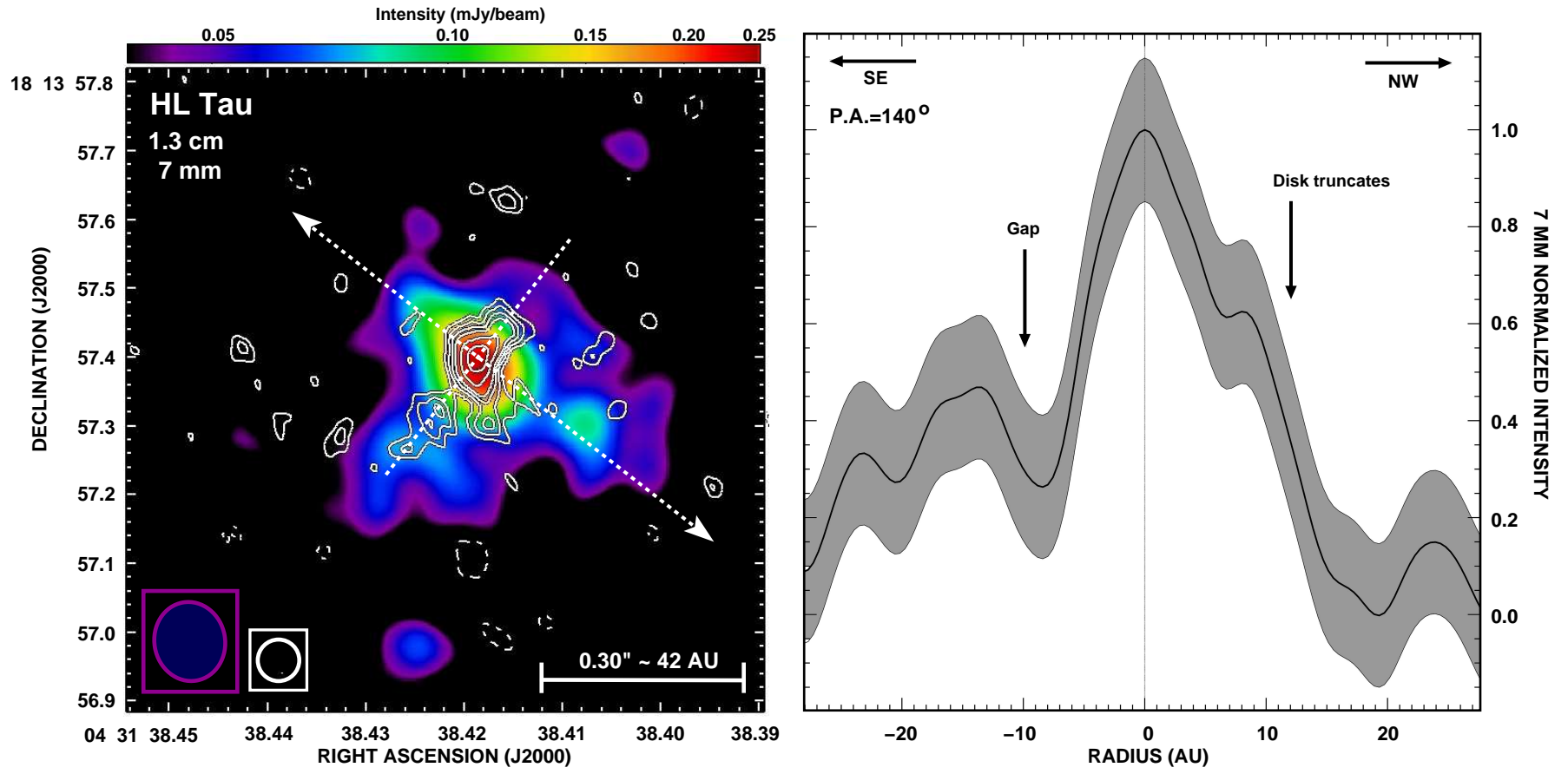

FIG. 1.- (a) Superposition of the $7 \mathrm{~mm}$ map (contours) over the $1.3 \mathrm{~cm}$ continuum map (color scale) of HL Tau. Contours at $7 \mathrm{~mm}$ are $-3,3,4,5,6,8,10$, $12,14,18,22,26$ and 32 times the rms of the map, $50 \mu \mathrm{Jy}$. The dashed lines mark the directions of the optical jet $\left(\mathrm{P} . \mathrm{A} .=50^{\circ}\right)$ and the disk $\left(\mathrm{P} . \mathrm{A} .=140^{\circ}\right)$. The half power contour of the $1.3 \mathrm{~cm}$ (filled ellipse) and $7 \mathrm{~mm}$ (empty ellipse) beams are shown in the bottom left corner. (b) A $7 \mathrm{~mm}$ intensity profile along the major axis of the disk (P.A. $=140^{\circ}$ ), obtained by averaging the emission at $\pm 0 !^{\prime \prime} 05$ from the axis marked in (a). The intensity error is indicated by the thickness of the profile. The disk truncates at a radius of $\sim 12 \mathrm{AU}$ to the NW, and shows a gap at a radius of $\sim 10 \mathrm{AU}$ to the SE. We speculate that these features could be related to the formation of a proto-planet (see text).

without significant detriment of the angular resolution. The $7 \mathrm{~mm}$ map of HL Tau shown here was made using data with baselines $>150 \mathrm{k} \lambda$ in order to resolve out extended emission that surrounds HL Tau. The nature of this extended emission is not fully understood, but probably originates from diffuse dust in the region. The $1.3 \mathrm{~cm}$ map of HL Tau shown in Greaves et al. (2008) was obtained from the same data, and, as expected, is very similar to our $1.3 \mathrm{~cm}$ map.

The rms noises of the natural weighting maps were $13 \mu \mathrm{Jy}$ at $1.3 \mathrm{~cm}$, and $50 \mu \mathrm{Jy}$ at $7 \mathrm{~mm}$. Synthesized beams are $\sim 0$ ! $^{\prime \prime} 10$ at $1.3 \mathrm{~cm}$ and $\sim 0 .^{\prime \prime} 05$ at $7 \mathrm{~mm}$. Bandwidth smearing at the position of XZ Tau degrades the resolution by $\sim 5 \%$ in the radial direction, which does not affect our analysis.

This region has a known proper motion of 0 ." $025 \pm 0$.'0035 $\mathrm{yr}^{-1}$ (Rodríguez et al. 2003), so we shifted the $20061.3 \mathrm{~cm}$ data by $0 .{ }^{\prime \prime} 04$ to align them with the $20047 \mathrm{~mm}$ data (with uncertainties of $0 .{ }^{\prime \prime} 005$ in proper motions and $0 .{ }^{\prime \prime} 01$ in absolute astrometry at each epoch).

\section{RESULTS AND DISCUSSION}

\subsection{HL Tau}

In Figure 19 we show a superposition of the $7 \mathrm{~mm}$ emission of HL Tau (contours) over the $1.3 \mathrm{~cm}$ map (colors). At 1.3 $\mathrm{cm}$, HL Tau shows a quadrupolar morphology consisting of two nearly perpendicular structures at position angles (P.A.s) of $\sim 50^{\circ}$ and $\sim 140^{\circ}$. As previously noted by Greaves et al. (2008), the alignment of the $1.3 \mathrm{~cm}$ emission at P.A. $\simeq 50^{\circ}$ with the optical jet of HL Tau (e.g. Anglada et al. 2007), suggests that the $1.3 \mathrm{~cm}$ emission at this P.A. is tracing freefree emission from the shock-ionized gas at the base of the flow. In contrast, the $1.3 \mathrm{~cm}$ emission at P.A. $\simeq 140^{\circ}$ is most probably tracing thermal dust emission from a circumstellar disk perpendicular to the outflow. This is more evident in our
$7 \mathrm{~mm}$ map (see Fig. 19), where the emission shows an elongated morphology with a P.A. of $\sim 140^{\circ}$ (perpendicular to the jet) and a size of $\sim 50 \mathrm{AU}$. The positions of the $1.3 \mathrm{~cm}$ and 7 mm maxima are nearly coincident and define the center of the radio jet.

We also detected two weak compact $1.3 \mathrm{~cm}$ features, one $\sim 0$." 4 to the NW (at a level of $3-\sigma$ ) and the other $\sim 0$." 4 to the $S$ (at a level of $4-\sigma$ ) of the $1.3 \mathrm{~cm}$ emission peak (see Fig. 11a). Greaves et al. (2008) identified the $1.3 \mathrm{~cm}$ NW feature with a $1.4 \mathrm{~mm}$ source detected by Welch et al. (2004), obtaining a spectral index of 2.5 in this wavelength range. They proposed that the $1.3 \mathrm{~cm} \mathrm{NW}$ feature is dust emission arising from a proto-planet with a mass of $14 \mathrm{M}_{J}$ and orbiting around HL Tau at a radius of $\sim 65 \mathrm{AU}$. The other $1.3 \mathrm{~cm}$ feature to the S, although somewhat stronger than the NW feature, was not discussed by these authors. As can be seen in Figure 1a, we have not detected $7 \mathrm{~mm}$ emission at the position of the proto-planet candidate, with a 3- $\sigma$ upper limit level of $\sim 0.15$ mJy. This upper limit and the $1.3 \mathrm{~cm}$ flux density of Greaves et al. (2008) implies a spectral index $<1$, suggesting free-free rather than dust emission. On the other hand, the spectral index of 2.5 between $1.3 \mathrm{~cm}$ and $1.4 \mathrm{~mm}$ obtained by Greaves et al. (2008) would imply a $7 \mathrm{~mm}$ flux density of $0.5 \mathrm{mJy}$, well above our 3- $\sigma$ upper limit. Additional, high angular resolution observations in the $\mathrm{mm}$ range are necessary to clarify the true nature of this source.

In Figure $1 \mathrm{~b}$ we show an intensity profile of the $7 \mathrm{~mm}$ emission along the major axis of the disk. As can be seen in Figures $1 \mathrm{l}$ and $1 \mathrm{~b}$, the $7 \mathrm{~mm}$ emission is knotty and asymmetric. The proposed disk extends $\sim 25$ AU to the SE, and shows a gap at a radius of $\sim 10 \mathrm{AU}$ and the emission to the NW appears truncated at a radius of $\sim 12-15$ AU. The gap and the truncation are probably related to a decrease of the density in 

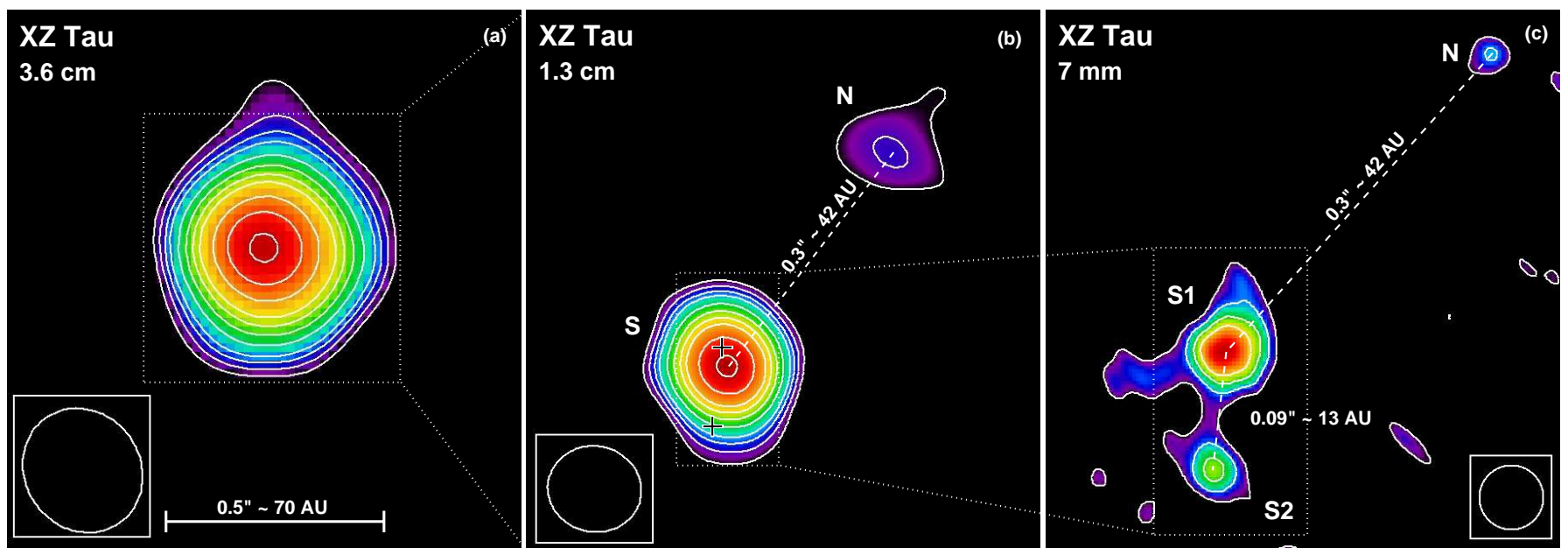

FIG. 2.- Radio continuum maps of XZ Tau at $3.6 \mathrm{~cm}$ (a), $1.3 \mathrm{~cm}$ (b) and $7 \mathrm{~mm}$ (c). The contours are -3, 3, 4, 5, 6, 8, 10, 12, 14, 18, 22 and 26 times the rms of each map, $7 \mu \mathrm{Jy}(3.6 \mathrm{~cm}), 13 \mu \mathrm{Jy}(1.3 \mathrm{~cm})$ and $50 \mu \mathrm{Jy}(7 \mathrm{~mm})$. At $3.6 \mathrm{~cm}$ a single radio source is detected. The $1.3 \mathrm{~cm}$ map shows two continuum sources, labeled as $\mathrm{N}$ and $\mathrm{S}$. The source $\mathrm{S}$ is marginally resolved, showing a slight elongation to the south. Crosses mark the positions of the two components $\mathrm{S} 1$ and $\mathrm{S} 2$ detected at $7 \mathrm{~mm}$. At $7 \mathrm{~mm}$, the source $\mathrm{N}$ is detected as a weak source, and the $1.3 \mathrm{~cm}$ source $\mathrm{S}$, is resolved in two components, $\mathrm{S} 1$ and $\mathrm{S} 2$.

TABLE 1

COMPONENTS OF THE XZ TAU TRIPLE SYSTEM

\begin{tabular}{|c|c|c|c|c|c|c|c|c|}
\hline \multirow{2}{*}{$\begin{array}{c}\text { Radio } \\
\text { Component }\end{array}$} & \multirow{2}{*}{$\begin{array}{c}\text { Optical } \\
\text { Component }\end{array}$} & \multicolumn{3}{|c|}{$\overline{\text { Position (J2000) }}{ }^{\mathrm{a}}$} & \multicolumn{2}{|c|}{ Flux Density $(\mathrm{mJy})^{\mathrm{a}}$} & \multirow{2}{*}{$\begin{array}{c}\text { Spectral } \\
\text { Index }\end{array}$} & \multirow{2}{*}{$\begin{array}{c}\text { Emission } \\
\text { Mechanism }\end{array}$} \\
\hline & & RA & DEC & Error & $1.3 \mathrm{~cm}$ & $7 \mathrm{~mm}$ & & \\
\hline $\mathrm{N}$ & XZ Tau B & 043140.0751 & 181357.079 & $0 .{ }^{\prime \prime} 006$ & $0.14 \pm 0.03$ & $0.3 \pm 0.2$ & $1.1 \pm 1.0$ & Free-free \\
\hline S1 & XZ Tau A & 043140.0891 & 181356.853 & $0 .{ }^{\prime \prime} 005$ & $0.33 \pm 0.03^{b}$ & $1.2 \pm 0.2$ & $1.9 \pm 0.3$ & Free-Free + Dust \\
\hline S2 & XZ Tau $C^{c}$ & 043140.0899 & 181356.762 & $0 .{ }^{\prime \prime} 008$ & $0.08 \pm 0.03^{b}$ & $0.8 \pm 0.2$ & $3.4 \pm 0.7$ & Dust \\
\hline
\end{tabular}

\footnotetext{
NOTES.- Units of right ascension are hours, minutes, and seconds, and units of declination are degrees, arcminutes, and arcseconds.

${ }^{a}$ Derived from Gaussian fits to the $7 \mathrm{~mm}$ natural weighting map (Epoch 2004.8). Errors in positions correspond to the relative positions. The error in the

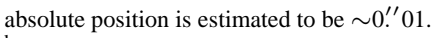

b Flux density derived by fitting the marginally resolved $1.3 \mathrm{~cm}$ source $\mathrm{S}$ to two unresolved Gaussian sources.

c This component is probably a deeply embedded object undetectable at optical/IR wavelengths.
}

the disk. Thus, since the disk is observed nearly edge-on (e.g. Cohen 1983), this gap could be associated with an annulus of low density in the disk with a radius of $\sim 10-15$ AU, similar to the radius of the orbit of Saturn, or twice the orbit of Jupiter. Such structures are expected to form as a consequence of the accretion of a planetary object forming in the disk (see, e.g. Bryden et al. 1999). Therefore, the gap and the truncation of the disk detected in our $7 \mathrm{~mm}$ map could be related to the formation of a proto-planet. Alternatively, this gap could be due to tidal truncation in a binary system (e.g., Pichardo et al. 2005) as in the case of HH30 (Anglada et al. 2007, Guilloteau et al. 2008). However, with the present data, these suggestions are very speculative, and should be confirmed by subsequent observations and/or modelling of the disk of HL Tau.

\subsection{XZ Tau}

In Figure $2 \mathrm{a}$ we show the $3.6 \mathrm{~cm}$ continuum map (angular resolution $\simeq 0$.' 3 ) of $\mathrm{XZ}$ Tau, obtained by Rodríguez et al. (1994), where a single radio continuum source was detected. Figures $2 \mathrm{~b}$ and $2 \mathrm{c}$ show our $1.3 \mathrm{~cm}$ and $7 \mathrm{~mm}$ maps, respectively. At $1.3 \mathrm{~cm}$ (angular resolution $\simeq 0$." 1 ), XZ Tau is resolved in two radio continuum sources (labeled $\mathrm{N}$ and $\mathrm{S}$ ) separated by $\sim 0$ ! $^{\prime} 3$ with a P.A. of $\sim 143^{\circ}$. The $7 \mathrm{~mm}$ map (angular resolution $\simeq 0$.'05), reveals that the $1.3 \mathrm{~cm}$ component $S$ is actually a double radio source (that we label as components S1 and S2) with a separation of $0 .^{\prime \prime} 09$ and a P.A. of $-6^{\circ}$.

The size of the synthesized beam of the $1.3 \mathrm{~cm}$ map $\left(\sim 0\right.$ ! $\left.^{\prime} 1\right)$, slightly larger than the separation between the S1 and S2 components at $7 \mathrm{~mm}\left(\sim 00^{\prime \prime} 09\right)$, does not allow to completely separate the emission of these components at $1.3 \mathrm{~cm}$. However, the $1.3 \mathrm{~cm}$ source $\mathrm{S}$ is marginally resolved in our map, showing a slight elongation to the south. In Figure $2 \mathrm{~b}$ we show the positions of the components $\mathrm{S} 1$ and $\mathrm{S} 2$ derived from the $7 \mathrm{~mm}$ map. As can be seen, most of the $1.3 \mathrm{~cm}$ emission seems to arise from component $\mathrm{S} 1$, since this is the source nearest to the peak of the $1.3 \mathrm{~cm}$ source $S$. The $1.3 \mathrm{~cm}$ source $\mathrm{S}$ can be fitted by two unresolved Gaussian sources separated by $\sim 0$." 08 , very similar to the separation between sources S1 and $\mathrm{S} 2$ in the $7 \mathrm{~mm}$ map.

In Table 1 we give the position (derived from the $7 \mathrm{~mm}$ map), flux density at $1.3 \mathrm{~cm}$ and $7 \mathrm{~mm}$, and the spectral index for each component. For source N, we obtained a spectral index of $1.1 \pm 1.0$ consistent with free-free emission from this source. For source S as a whole, we obtained a "combined" spectral index of $2.5 \pm 0.3$ (obtained from the total flux density of the $1.3 \mathrm{~cm}$ source $\mathrm{S}$, and the sum of the $7 \mathrm{~mm}$ flux densities of $\mathrm{S} 1$ and $\mathrm{S} 2$ ). This steep spectral index suggests a combination of free-free plus thermal dust emission. If we use the flux densities obtained from a double Gaussian fit to the $1.3 \mathrm{~cm}$ source $\mathrm{S}$, for source $\mathrm{S} 1$ we obtain a spectral index of $1.9 \pm 0.3$ which suggests a combination of free-free plus thermal dust emission. In contrast, for S2 we obtain a spectral index of $3.4 \pm 0.7$ which suggests that the emission of this source is dominated by thermal dust emission. 

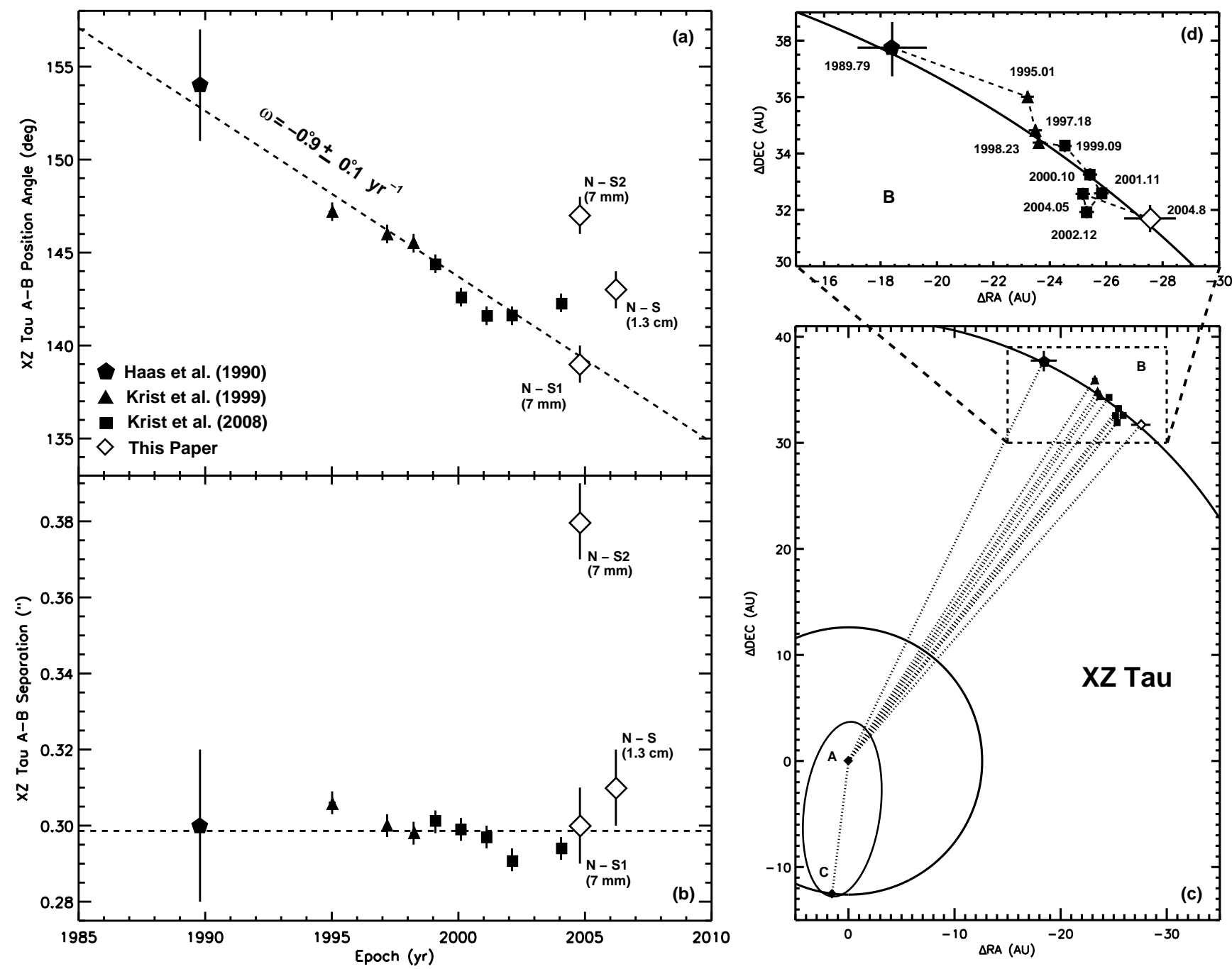

FIG. 3. - (a) Plot of the P.A. between the optical/IR components XZ Tau A and XZ Tau B vs. time (Haas et al. 1990; Krist et al 1999, 2008). The dashed line is a least squares fit to these data, and corresponds to an orbital angular velocity of $-0.9 \pm 0.1$ degrees $\mathrm{yr}^{-1}$. Three different P.A.s measured from our $1.3 \mathrm{~cm}$ and $7 \mathrm{~mm}$ maps are marked with diamonds. Only the P.A. between the $7 \mathrm{~mm}$ sources $\mathrm{N}$ and $\mathrm{S} 1$ seems to be compatible with the angular orbital velocity derived from the optical/IR data. This suggests that the source S1 is the radio counterpart of the optical component XZ Tau A. (b) Plot of the separation between the optical/IR components XZ Tau A and XZ Tau B vs time. The measurements are compatible with a constant separation of 0 .' $30 \pm 0$.' 01 . (c) and (d) A schematic representation of the XZ Tau triple system. The positions of XZ Tau B relative to XZ Tau A, derived from the P.A. and separation measurements of Haas et al. (1990), Krist et al $(1999,2008)$ and our data, are shown. The current data are compatible with a circular face-on orbit of XZ Tau B relative to XZ Tau A. In contrast, with only a single detection of the third component, XZ Tau C, we have no information about its orbital motion. Two hypothetical orbits (circular and elliptical) for XZ Tau C are shown.

As mentioned above, $\mathrm{XZ}$ Tau is known to be an optical/IR

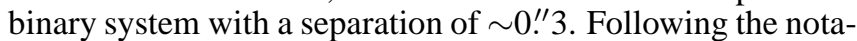
tion of Krist et al. (2008), we will refer to the optical components of this system as XZ Tau A (the southern optical component) and XZ Tau B (the northern optical component). Krist et al. $(1999,2008)$ observed XZ Tau with the HST in several epochs, and detected a change in the P.A. of the binary due to orbital motions. According to this motion $\left(\sim-0.9{\left.\mathrm{deg} \mathrm{yr}^{-1}\right)}^{-1}\right.$ at the epoch of our radio observations, component XZ Tau B must be still located north of component XZ Tau A. Therefore, we identify the radio source $\mathrm{N}$ with the optical component XZ Tau B.

The identification of the two radio sources at the south (S1 and S2) with the other optical component (XZ Tau A) is not straightforward. One possibility is that both radio sources have optical emission that could not be resolved by the HST observations of Krist et al. (2008). However, another possibility is that only one of the radio sources is the counterpart of the optical component. In order to investigate these possibilities, we have plotted in Figures $3 \mathrm{a}$ and $3 \mathrm{~b}$ the P.A. and separation of the optical binary measured in each epoch together with the P.A.s and separations between the different radio components measured from our $1.3 \mathrm{~cm}$ and $7 \mathrm{~mm}$ maps. As can be seen, the P.A. and separation between the radio sources $\mathrm{N}$ and $\mathrm{S} 1$ seems to be the only one compatible with the previous optical/IR data. This suggests radio source $\mathrm{S} 1$ as the sole counterpart to the optical component XZ Tau A; $\mathrm{S} 2$ is optically undetected. This is similar to the case of the close binary SVS 13, where both components are detected at $\mathrm{cm}$ wavelengths, but only one object is detected in the optical regime (Anglada et al. 2004). As we have discussed above, the radio emission of component $\mathrm{S} 2$ seems to be dominated 
by thermal dust emission. We believe that this source traces a star since if it not were gravitationally bound, it would quickly dissipate. The size of Sb is smaller than $7 \mathrm{AU}$, that expanding at only $1 \mathrm{~km} \mathrm{~s}^{-1}$, will dissipate in only 30 years. Then, we conclude that this dust must be gravitationally bound to a star and it is most possibly an envelope or a disk. This suggests that source S2 is a more deeply embedded object so it is not surprising that it is optically very faint. Therefore, we propose that the radio source S2 is an optically invisible third component of the XZ Tau system. For consistency with the previous nomenclature, we call this component XZ Tau C.

In summary, the results derived from our $1.3 \mathrm{~cm}$ and $7 \mathrm{~mm}$ maps suggest that XZ Tau, previously known as a binary system, is actually a triple star-system (see Figs. 35 and 3 d). The previously known optical/IR components, XZ Tau A and $\mathrm{XZ}$ Tau $\mathrm{B}$, have $1.3 \mathrm{~cm}$ and $7 \mathrm{~mm}$ counterparts and are separated by $42 \mathrm{AU}$. A third component, $\mathrm{XZ}$ Tau $\mathrm{C}$ has been detected for the first time in our $1.3 \mathrm{~cm}$ and $7 \mathrm{~mm}$ maps, and is found to be a more deeply embedded object, probably undetectable at optical/IR wavelengths, that forms a close binary system with XZ Tau A with a separation of only 13 AU.

Each optical component of the XZ Tau system (A and B) is known to drive a collimated jet (Krist et al 2008). It has been proposed that the jet of XZ Tau A underwent a large velocity pulse approximately in 1980 (Krist et al. 2008) which, after shocking and heating the gas near the star, would result in the $70 \mathrm{~km} \mathrm{~s}^{-1}$ expanding bubble detected in the series of HST images of Krist et al. (1999, 2008). These authors explored the possibility that this ejection was triggered by the passage of component XZ Tau B very near component XZ Tau A. However, even assuming a very eccentric orbit (e $\simeq 0.9)$ they derived that the last approach of these components would have taken place in 1955, and thus, they discarded this possibility.

The discovery of a new companion, closer to XZ Tau A, suggests that it is once more feasible to consider the passage of this companion as the ejection trigger. If we assume a faceon, nearly circular $(\mathrm{e} \simeq 0.1)$ orbit and a total mass of $1 \mathrm{M}_{\odot}$ (as suggested by the results of Krist et al. 1999 and White \& Ghez 2001), we derive an orbital period of $\sim 40 \mathrm{yr}$. If at the time of our $7 \mathrm{~mm}$ observations (2004.8) the XZ Tau A$\mathrm{C}$ system wass near apoastron, then the last periastron passage must have taken place one half-period before, i.e. nearly 1980, the date estimated for the ejection. Further observations are needed to produce a more accurate ephemeris for the XZ Tau system. The present results predict a new periastron passage around 2020, and then a new major ejection from $\mathrm{XZ}$ Tau A at this epoch.

Acknowledgements. We thank A.M.S. Richards for valuable comments that improved the paper. C.C.-G. acknowledges support from MEC (Spain) FPU fellowship. G.A. and C.C.-G. acknowledge support from MEC (Spain) AYA200508523-C03-03 and MICINN (Spain) AYA2008-06189-C0301 grants (co-funded with FEDER funds), and from Junta de Andalucía (Spain). L.F.R. and S.C. acknowledge the support of DGAPA, UNAM, and CONACyT (México).

\section{REFERENCES}

Anglada, G., López, R., Estalella, R., Masegosa, J., Riera, A., \& Raga, A. C. 2007, AJ, 133, 2799

Anglada, G., Rodríguez, L.F., Osorio, M., Torrelles, José M., Estalella, R., Beltrán, M.T., Ho, P.T.P. 2004, ApJ, 605, L137

Bryden, G., Chen, X., Lin, D. N. C., Nelson, R. P., \& Papaloizou, J. C. B. 1999, ApJ, 514, 344

Cohen, M. 1983, ApJ, 270, L69

Greaves, J. S., Richards, A. M. S., Rice, W. K. M., \& Muxlow, T. W. B. 2008 , MNRAS, 391, L74

Guilloteau, S., Dutrey, A., Pety, J., Gueth, F. 2008, A\&A, 478, 31

Haas, M., Leinert, C., \& Zinnecker, H. 1990, A\&A, 230, L1

Kenyon, S. J., Dobrzycka, D., \& Hartmann, L. 1994, AJ, 108, 1872

Krist, J. E., et al. 1999, ApJ, 515, L35

Krist, J. E., Stapelfeldt, K. R., Hester, J. J., Healy, K., Dwyer, S. J., \& Gardner, C. L. 2008, AJ, 136, 1980

Looney, L. W., Mundy, L. G., \& Welch, W. J. 2000, ApJ, 529, 477

Monin, J.-L., Pudritz, R. E., \& Lazareff, B. 1996, A\&A, 305, 572
Mundt, R., Ray, T. P., Bührke, T., Raga, A. C., \& Solf, J. 1990, A\&A, 232, 37 Mundt, R., \& Fried, J. W. 1983, ApJ, 274, L83

Pichardo, B., Sparke, L.S., Aguilar, L.A. 2005, MNRAS, 359, 521

Rodríguez, L. F., Cantó, J., Torrelles, J. M., Gómez, J. F., Anglada, G., \& Ho, P. T. P. 1994, ApJ, 427, L103

Rodríguez, L.F., Curiel, S., Cantó, J., Loinard, L., Raga, A.C.; Torrelles, J.M. 2003, ApJ, 583, 330

Sargent, A. I., \& Beckwith, S. V. W. 1991, ApJ, 382, L31

Torrelles, J. M., Anglada, G., Rodríguez, L. F., Cantó, L. F., \& Barral, J. F. 1987, A\&A, 177, 171

Torres, R.M., Loinard, L., Mioduszewki, A.J., \& Rodríguez, L.F. 2009 submitted to ApJ.

Wilner, D. J., Ho, P. T. P., \& Rodríguez, L. F. 1996, ApJ, 470, L117

White, R. J., \& Ghez, A. M. 2001, ApJ, 556, 265

Welch, W. J., Webster, Z., Mundy, L., Volgenau, N., \& Looney, L. 2004, in Bioastronomy 2002, IAU Symp. 213, eds. R. Norris, F. Stootman (ASP, San Francisco), p59 